\title{
A ground reaction force analysis in walking and running gaits in horse leg model on viscoelastic hoof-ground contact
}

\author{
Dondogjamts Batbaatar \\ Graduate School of Life Science and Systems Engineering, Kyushu Institute of Technology, 2-4 Hibikino, Wakamatsu-ku, \\ Kitakyushu 808-0196, Japan \\ Hiroaki Wagatsuma \\ Graduate School of Life Science and Systems Engineering, Kyushu Institute of Technology, 2-4 Hibikino, Wakamatsu-ku, \\ Kitakyushu 808-0196, Japan \\ RIKEN Center for Brain Science, Saitama, Japan \\ E-mail: batbaatar.dondogjamts581@mail.kyutech.jp,waga@brain.kyutech.ac.jp \\ www.kyutech.ac.jp
}

\begin{abstract}
In this paper, we focused on the hoof-ground interaction in the simplified horse leg model because walking and running gaits are known to be different in trajectories; however, the force analysis still remains as unsolved issues. The computational experiments in Matlab, elastic and inelastic impact with the ground was resolved by using the dissipative contact force model and the ground reaction force was clearly examined in four different conditions from the combination of walking/running and elastic/inelastic contact.
\end{abstract}

Keywords: Closed-loop linkage; Multibody dynamics; Dissipative contact force model; hoof-ground interaction.

\section{Introduction}

In the human gait analysis, three different foot-rocker mechanisms are known as heel rocker, ankle rocker and forefoot rocker, which are generated from pendulum dynamics with three fulcrum points of heel, ankle and toe [1]. In a gait cycle, there are two phases as swing stance phases and particularly in the human gait analysis, the stance phase is decomposed to the initial contact for touching with the ground from the heel, the loading response for shifting of the center of mass from back to front, the mid-stance for lifting the heel up, the terminal stance for transition to the swing phase, and then preswing [2]. Such as bipedal gait cycle can be simplified to a mechanical system based on a coupled pendulum as demonstrated by McGeer (1990) [3], called a passive dynamic walking robot without any electromechanical actuator to mimic three rockers. In this case, the compliant human leg dynamics has been studied with a series of contact points on the rolling surface [4-6] and with the effective roll-over geometry of leg motion [7-9]. The fact suggests that the utilization of the simple skeleton model provides a large benefit to compensate the limitation of experimental measurements in the gain analysis to realize the actual phenomenon of the ground reaction force, because there are evidences in the walking condition $[10,11]$, such as demonstrated two peaks in the temporal evolution of the ground reaction force during the stance phase, while there is a lack of evidences in other gait patterns. In robotic applications inspired from biological mechanisms, an articulated leg with hoof was inspired from the horse [12] and a linkage mechanism to mimic the horse leg trajectory by Batbaatar \& Wagatsuma (2019) [13] and similar approaches were found in closed-loop linkage mechanisms $[14,15]$. In the animal locomotion, there are various gait patterns known 
as walk, trot, gallop and so on [16]. Therefore, the establishment of the detail analytical method for the ground reaction force is highly important to elucidate the principle to differ gaits in the sense of the energy consumption. Focusing on the ground contact phenomenon, the hoof part of the horse leg mechanism can be modelled in the form of the triangle structure to connect three representative points as the toe, heel and ankle, which reproduces the rocker mechanisms. If it is possible to provide kinematic/kinetics of the hoof-ground contact, the viscoelastic effect is also considerable to analyse how much the leg absorbs the impact force when touching with the ground. It realizes the detail analysis of the amount of the energy storage discussed in the distal leg movement and landing motions $[17,18]$. The purpose of the present study is to establish the basement analytical method for the ground reaction forces in gaits and demonstrate the efficacy of the method in the simplified leg model to focus on the hoof-ground interaction. This paper is divided into following sections. Section 2 introduces the general Multibody dynamics (MBD) formulation applied to the proposed leg mechanism as well as the viscoelastic contact force introduced in order to simulate realistic hoof-ground contact. Section 3 contains the kinematic and kinetic analysis focusing on the hoof-ground interaction, while Section 4 summarizes the main results and discusses the potential improvements and limitation of the study.

\section{Methods}

\subsection{Multibody system formulation for horse leg mechanism}

Kinematics and dynamic analysis of horse leg mechanism with multibody dynamics was presented in the article by Batbaatar \& Wagatsuma (2019) [13] in which simplified model of leg mechanism generated a flexible coordinated movement of hoof with constraint closed-loop linkage structure. Furthermore, in applying appropriate driver constraint in system as principle movement strategy in running animal functional and animal-like end trajectories were generated and evaluated with duty factor which defines the specific locomotor behavior with respect to the stance and swing timing in one locomotive cycle. Mathematical model for horse leg mechanism can be stated by using multibody dynamics (MBD), the vector $\boldsymbol{q}$ with 42 elements including position and orientation of each body in leg mechanism expressed as generalized coordinates can be written

$$
\boldsymbol{q}=\left[q_{1}^{T}, q_{2}^{T}, q_{3}^{T}, \ldots, q_{14}^{T}\right]^{T} .
$$

The vector of $q$ contains $42(=14 \times 3)$ elements which are 14 rigid links and their center of position $x_{i}, y_{i}$ and orientation $\phi_{i}$ are obtained in the generalized coordinates in the present analysis. A set of kinematic algebraic constraint equation according to the given initial configuration can be written

$$
\Phi(q, t)=\left[\begin{array}{c}
\Phi^{K}(q) \\
\Phi^{D}(q, t)
\end{array}\right]_{42 \times 1}=0 .
$$

where the first 41 elements of the column matrix of kinematic constraint equation $\Phi^{K}(q)$ are derived from the absolute constraints between body and fixed ground node. The last element $\Phi^{D}(q, t)$ defines the driver constraint (Crankshaft) of the proposed leg mechanism. The partial derivative of kinematic constraint equation respect to the generalized absolute Cartesian coordinates $\boldsymbol{q}$ is Jacobian matrix $\boldsymbol{\Phi}_{q}$ is obtained as

$$
\boldsymbol{\Phi}_{q}=\left[\frac{\partial \Phi(\boldsymbol{q}, t)}{\partial \boldsymbol{q}}\right]_{42 \times 42},
$$

where it allows us to investigate placement, velocity and acceleration analyses kinematically. The forward dynamics analysis introduces the mass matrix $\mathbf{M}=(42 \times 42)$, and the generalized external force vector $\mathbf{h}^{(\mathbf{a})}=(42 \times 1)$, as follows:

$$
\begin{gathered}
\mathbf{M}=\operatorname{diag}\left(M_{1}, M_{2}, \ldots, M_{14}\right), \\
\left\{\mathbf{M}_{i}=\left[m_{i}, m_{i}, J_{i}\right]^{T} \mid i=1,2, \ldots, 14\right\}, \\
\mathbf{h}^{(\mathbf{a})}=\left[h_{1}^{(a)^{T}}, h_{2}^{(a)^{T}}, \ldots, h_{14}^{(a)^{T}}\right]^{T} \\
\left\{\mathbf{h}_{\mathbf{i}}^{(\mathbf{a})}=\left[0,-m_{i} g, 0\right]^{T} \mid i=1,2, \ldots, 14\right\},
\end{gathered}
$$

where $m_{i}$ is the mass of rigid link to point $i, J_{i}=2 l_{i} / 3$ is the polar moment of inertia of rigid link to point $i$, and $g$ is the gravitational acceleration. The equation motion of the system for the computer system analysis can be is expressed in general matrix form as

$$
\left[\begin{array}{cc}
\boldsymbol{M} & \boldsymbol{\Phi}_{q}^{\boldsymbol{T}} \\
\boldsymbol{\Phi}_{\boldsymbol{q}} & \mathbf{0}
\end{array}\right]\left[\begin{array}{l}
\ddot{\boldsymbol{q}} \\
\lambda
\end{array}\right]=\left[\begin{array}{c}
\boldsymbol{h}^{(a)} \\
\gamma-2 \alpha \dot{\boldsymbol{\Phi}}-\beta^{2} \boldsymbol{\Phi}
\end{array}\right] .
$$


where $\ddot{q}$ is the acceleration vector, $h^{(a)}$ denotes the generalized external force vector, $\lambda$ is the Lagrange multipliers with

vector $\Phi_{q} \ddot{\boldsymbol{q}}=-\left(\boldsymbol{\Phi}_{q} \dot{\boldsymbol{q}}\right)_{q} \dot{\boldsymbol{q}}-2 \boldsymbol{\Phi}_{\boldsymbol{q} t} \dot{\boldsymbol{q}}-\boldsymbol{\Phi}_{t t}=\gamma$. The parameter $\alpha$ and $\beta$ are the feedback compensator in Baumgarte stabilization method [19] used to guarantee accuracy of the numerical solution. In the forward dynamic analysis, new coordinates and velocities require two arrays for $\dot{\boldsymbol{q}}$ and $\ddot{\boldsymbol{q}}$ for the time step $t+\Delta t$ as

$$
\boldsymbol{u}=\left[\begin{array}{c}
\boldsymbol{q} \\
\dot{\boldsymbol{q}}
\end{array}\right], \quad \dot{\boldsymbol{u}}=\left[\begin{array}{c}
\dot{\boldsymbol{q}} \\
\ddot{\boldsymbol{q}}
\end{array}\right], \quad \dot{\boldsymbol{u}} \stackrel{\text { yields }}{\longrightarrow} \boldsymbol{u}(t+\Delta t),
$$

At the starting point of the numerical simulation, initial configurations of target mechanisms are given according to the primary operation in forward dynamics analysis.

\subsection{Formulation of the contact-impact process in planar multibody system}

When describing the contact impact event between the hoof and ground, we need to determine the state of the contact and accurately acquire the relative deformation and speed at the instant of contact.

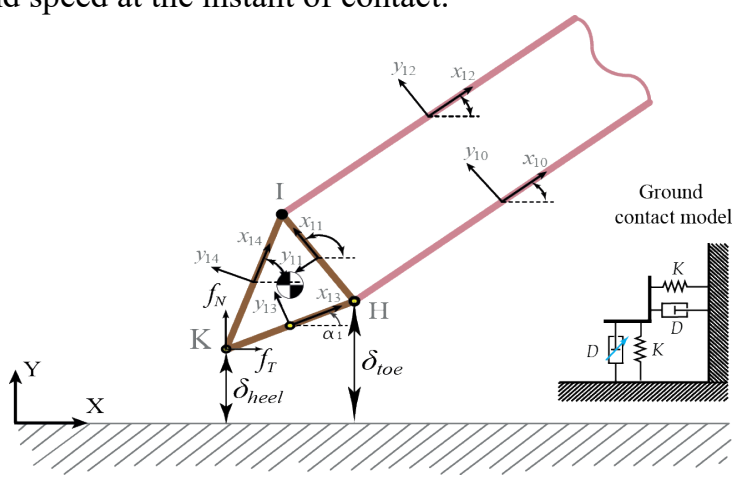

Fig. 1. Representations of foot-ground interactions by kinematic model of hoof-ground contact.

As shown in Fig. 1, only non-contact scenarios is considered in the kinematic model. When the leg contacts with the ground, the deformation or penetration is estimated as

$$
\delta=y_{G}-P_{n},
$$

where the $P_{n}$ is the normal component of the position vector or the potential contact point as $K=\left[K_{x}, K_{y}\right]$ (toe-first) or $H=\left[H_{x}, H_{y}\right]$ for the case of heel first contact. The ground plane $y_{G}$ is consistently levelled from the lowest point of the locomotive trajectory in the sense of the relative indentation.

$$
\delta=\left\{\begin{array}{ccc}
\delta & (\delta>0 & \left.F_{N}=0\right) \\
-\delta & (\delta \leq 0 & \left.F_{N} \geq 0\right)
\end{array}\right.
$$

In Eq. 11, the positive value of $\delta$ is that distance represent a separation, while negative values denote relative penetration of the contacting bodies. Therefore, the sign of penetration indicates the phase transition from swing to stance and vice versa for the case of hoofground contact. By using Eq. 10, it is clear to discriminate walking phases and contact forces as continuous function of deformation. Based on updated position and velocity of potential contact points on the hoof $K=\left[K_{x}, K_{y}\right]$ or $H=\left[H_{x}, H_{y}\right]$ from the multibody kinematic analysis, relative normal and tangential velocity among the contact points are estimated as

$$
v_{n}=\dot{\delta}=\left(\dot{r}_{j}-\dot{r}_{i}\right)^{T} n,
$$

similarly,

$$
v_{t}=\left(\dot{r}_{j}-\dot{r}_{i}\right)^{T} t
$$

where $t$ is the unit vector in tangential direction obtained by rotating unit normal vector $n$ counter-clockwise by 90 degrees. When bodies come into contact, the normal contact force can be estimated with contact force model introduced by Lankarani \& Nikravesh (1990) [20]. In the damping factor model, the crucial parameter known as coefficient of restitution was introduced which defines the whether contact is fully elastic $c r=1$ (or plastic) $c r=0$, resulting impact force model can be written

$$
F_{N}=K \delta^{n}\left[1+\frac{3\left(1-c r^{2}\right)}{4} \frac{\dot{\delta}}{\dot{\delta}^{(-)}}\right] .
$$

where $\mathrm{cr}$ denotes the coefficient of restitution which is defined as the ratio of relative approach velocity $\dot{\delta}^{(+)}$ and relative departing velocity of $\dot{\delta}^{(-)}$. The tangential friction force in contact is calculated using modified Coulomb friction law [21]. The dynamic friction forces $F_{f}$ in the presence of sliding [22] can be written as

$$
F_{f}=-\mu_{k} F_{N} c_{f} c_{d}
$$


where $\mu_{k}$ the kinetic friction coefficient. In order to take the friction force effect into account, the direction of the tangential velocity vector $c_{f}=\left|v_{T}\right|$ with the target body velocity $v_{T}$, which is opposite to direction of motion. $c_{d}$ is the dynamic correction coefficient given by [21]. If the normal force $F_{N}$ is obtained from a contact with a forcedeformation (Eq. 14.) of contact force model, a logical point-to-point spring-damper element [23] is only active during the period of contact in normal and tangential direction as shown in kinematic model in Fig. 1. Finally, absolute values of the force vector in normal and tangential defines the resultant ground reaction forces $G R F=\sqrt{F_{N}+F_{f}}$ exerted when the hoof press against the ground.

\section{Results}

According to the MBD formulation above, the constitutive contact force model is calculated numerically with kinematic and dynamic analyses, which allow to visualize temporal evolutions of the locomotive trajectory, resultant ground reaction force of the leg mechanism.

Table 1. Parameters used in the numerical simulation.

\begin{tabular}{lcc}
\hline \multicolumn{3}{c}{ Kinematic/Dynamic analysis } \\
\hline $\begin{array}{l}\text { Gravitational acceleration } \\
{\left[\mathrm{m} / \mathrm{s}^{2}\right]}\end{array}$ & $g$ & 9.81 \\
$\begin{array}{l}\text { The velocity of the driving } \\
\text { crank [rad/s] }\end{array}$ & $\omega$ & $2 \pi$ \\
Total simulation time $[\mathrm{s}]$ & $t$ & $0 \leq \mathrm{t} \leq 1$ \\
Baumgarte parameter & $\alpha$ & 15 \\
Baumgarte parameter & $\beta$ & $\sqrt{2 \alpha}$ \\
Time step [s] Contact force analysis & $K$ & $1.0 \times 10^{-3}$ \\
\hline \multicolumn{3}{c}{$\quad c r$} \\
\hline Stiffness [N/m] & $K$ & $0 \leq c r \leq 10^{4}$ \\
Coefficient of restitution & $c r$ & 0.4 \\
Coefficient of kinetic friction & $\mu_{k}$ & \\
\hline
\end{tabular}

Table 1 displays the parameters used in the numerical simulation for contact force analyses of horse leg mechanisms. Matlab-based numerical simulation was performed with a combination of the Euler method with the time step of $1 \times 10^{-3} \mathrm{~s}$. For the contact analysis to prevent an unnatural rise of ground reaction force, the stiffness is no smaller than $2 \times 10^{4} \mathrm{~N} / \mathrm{m}$ for the contacting bodies is considered according to simulation in [24]. It's simply that the ground deformation is equivalent to the resultant motion of the stiffness-damping system under the compression. Two trajectories were selected as typical trajectories to reproduce walking and running gaits according to the mechanics of the simple model, which are generated by changing the control parameters associated with driver constraint in the horse leg mechanism.

As shown in Fig.2, the $1^{\text {st }}$ trajectory considered to be a walking in term of duty factor and step length, the relation was evaluated in previous study. The $2^{\text {nd }}$ $1^{\text {st }}$ trajectory

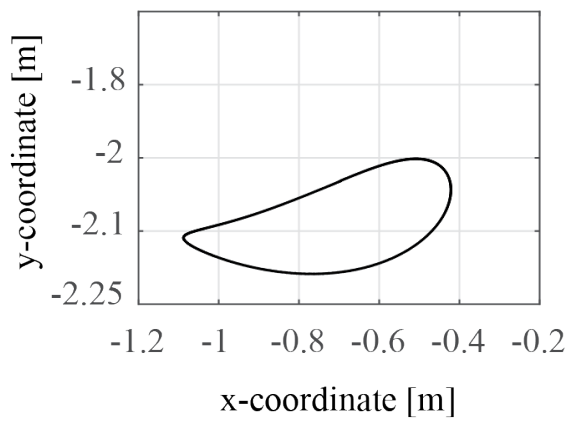

(a)

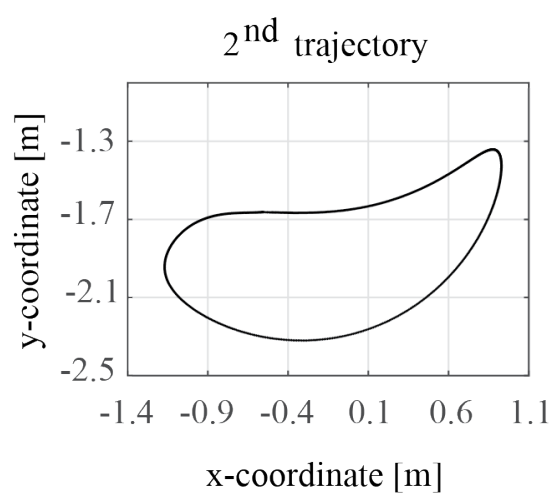

(b)

Fig. 2. Gait trajectories generated from the horse leg motion. Walking (a), Running (b).

trajectory which has longer step length compared to the $1^{\text {st }}$ trajectory considered to be running gait in which leg orientation controlled by swinging motion with respect to the body, which may potentially generate the propulsive ground reaction force due the intrinsic high rate of angular oscillation at the hoof.

In the temporal evaluation of the hoof angle as shown in Fig. 3, walking and running gaits were generated in accordance with the first and second trajectories in Fig. 

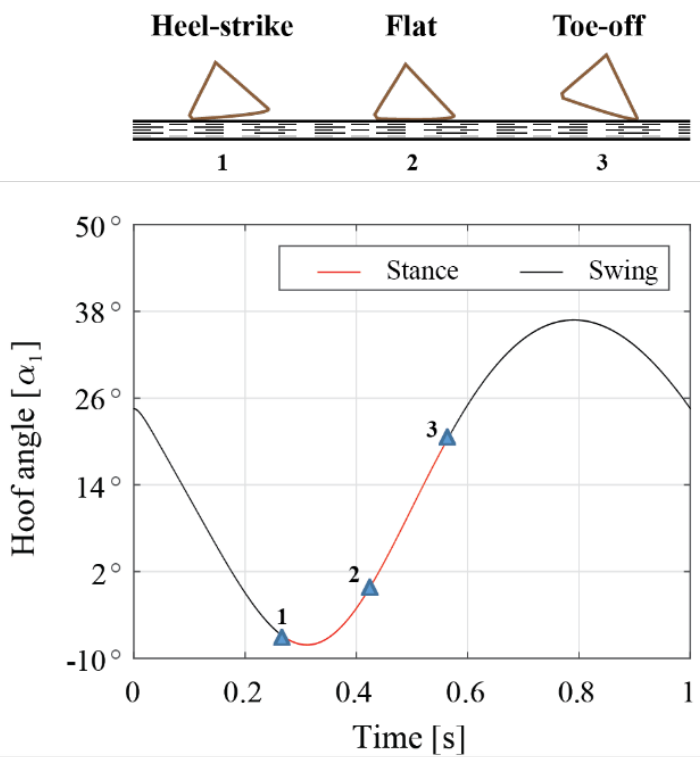

(a)
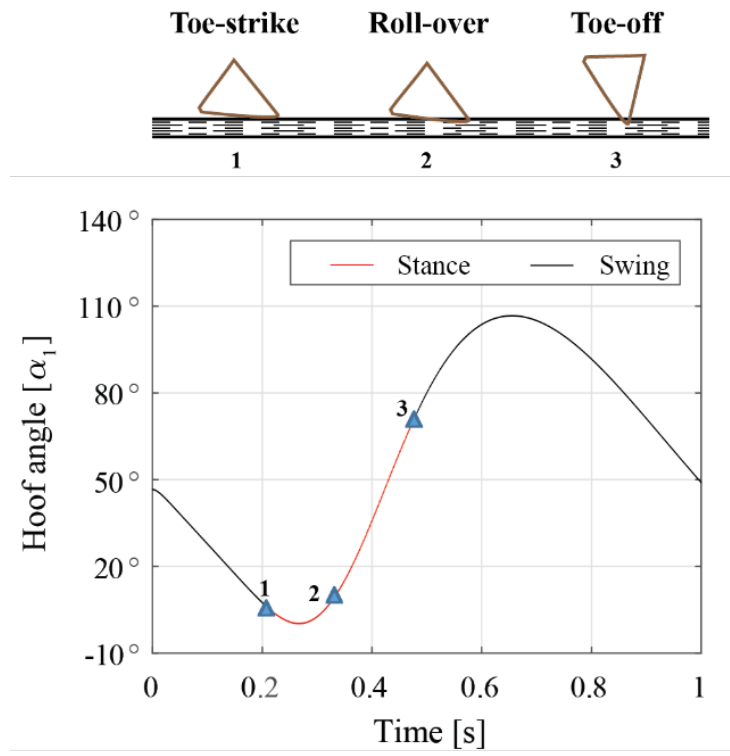

(b)

Fig. 3. Hoof angle variations with respect to the locomotive trajectory. Angular rotation of hoof in one locomotive cycle of walking gait (a) and running gait (b).

2. In the waling gait, the transition of sub-phases commonly occurred in the rolling motion of the triangle structure in both cases. In the walking gait, those subphases from heel-strike, flat and toe-off were gradually generated (Fig. 3(a). top), which is consistent with the gradual change of the hoof angle (Fig.3 (b). top), while in the running gait, the transition started from the toestrike that causes the roll-over sub-phase quickly and then reached to the toe-off sub-phase with a large gap from the ground rather than that in the walking gait. The result was numerically examined as the first contact occurs at instant time $t_{1}=0.267 \mathrm{~s}$ and $\alpha_{1}=-6.83^{\circ}$ leaves the ground at $t_{3}=0.569 \mathrm{~s}$ and $\alpha_{1}=-20.9^{\circ}$ for the $1^{\text {st }}$ (walking) trajectory. In the case, the hoof rolling over the ground and during the mid-stance multiple contact points were examined at $\alpha_{1}=-0.23^{\circ}$ degree in which hoof was almost parallel with the ground. When the hoof leaves the ground, angular variation during the contact phase was reached $26^{\circ}$ degrees. For the $2^{\text {nd }}$ (running) trajectory, the contact phenomenon occurred at the instant time $t_{1}=0.208 \mathrm{~s}$ and $\alpha_{1}=6.20^{\circ}$ firstly, shifted to leaving from the ground at $t_{3}=0.478 \mathrm{~s}$ and $\alpha_{1}=71.08^{\circ}$. Finally, the hoof angle was reached $64.8^{\circ}$ degrees, which is approximately 2.5 times larger than the result in the walking gait. Interestingly, the hoof-ground interaction was significantly influenced by the initial impact phenomenon, which differentiate the grounding part either heel or toe and it reflects to successive sub-phases too. Even in the simple model, the differentiation was clearly observed not only in the trajectory level as a posture and kinematic analysis but also in the kinetic level as the dynamics analysis, which is easily extended to the energy analysis. All these sub-phases are characterized as representative sub-phase to reconstruct the target gait and it can provide the parametric analysis with stiffness and damping, which is associated with an actual parameter from soft tissues in the distal limb. It is because that the compliant contact force model was theoretically described as shown in the method section, which allow to change those parameters related to reaction forces easily.

In the Matlab based numerical simulation, ground reaction forces were evaluated by using Lankarani \& Nikravesh [20] model by changing restitution coefficients as $c r=1$ for elastic and $c r=0$ for inelastic contact cases. According to the analysis, the maximum contact force was obtained as $453.65 \mathrm{~N}$ for the $1^{\text {st }}$ (walking) trajectory in the inelastic contact condition and $175.34 \mathrm{~N}$ in the elastic case. Average normal force was $80.83 \mathrm{~N}$ and $34.88 \mathrm{~N}$ respectively. For the $2^{\text {nd }}$ (running) trajectory, the maximum GRF was $8.694 \mathrm{kN}$ for the 


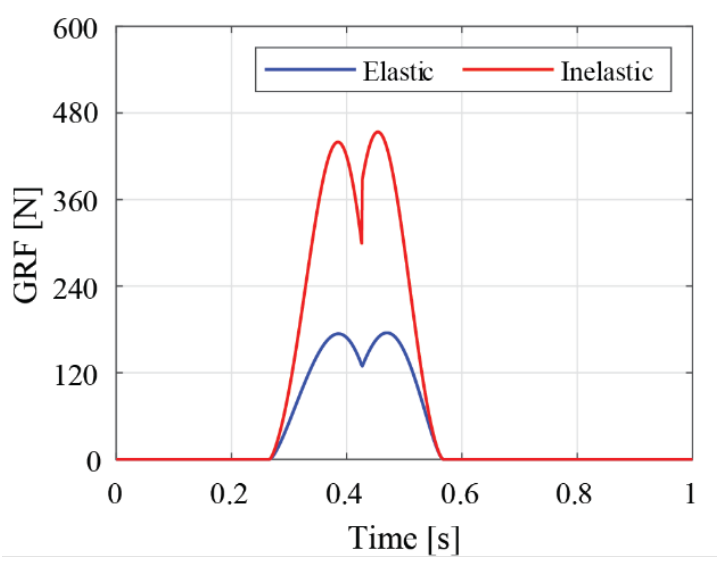

(a)

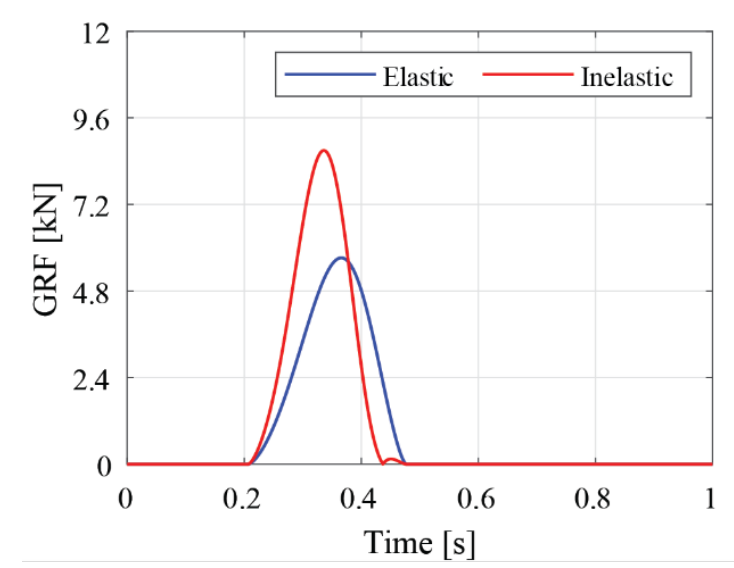

(b)

Fig. 4. Resultant values of ground reaction forces under the different contact condition. Force pattern of walking gait (a) and running gait (b).

inelastic contact condition and $5.719 \mathrm{kN}$ in the elastic case. Average normal force was $978 \mathrm{~N}$ and $800 \mathrm{~N}$ respectively. Result showed that the significant reduction of ground reaction force with respect to the viscoelastic contact was estimated as $61.35 \%$ in $1^{\text {st }}$ trajectory and $34.22 \%$ in $2^{\text {nd }}$ trajectory. In the walking trajectory, multiple point support occurring in which hoof loaded the body weight at mid-stance as shown in Fig. 4(a), the maximum pressure or force peak indicated in only when the leg first impact and leaves the ground which was consistent with the force pattern observed in the human walking gait $[25,26]$.

\section{Conclusion and Discussion}

For the sake of the establishment of the generalized analysis of the viscoelastic effect with respect to various gait patterns, we introduced a novel theoretical method as the integrative framework of the multibody dynamics and contact force model and then the computational analysis clearly demonstrated results in four different conditions from the combination of walking/running and elastic/inelastic contact. In the simplification of the hoof mechanism by using a triangle structure to focus on three nodes as heel, ankle and toe, the result revealed not only the two peak generation in the temporal evolution of the ground reaction force during the stance phase of the walking gait, but also a single peak observation in the case of the running gait. The phenomenon was generated from that wheel-like rolling motion of the hoof support and stabilizes the body in mid-stance, and partially consistent with the observation in the human foot-ground interaction $[1,10]$. In spite of the existence of the complexity in the horse leg structure, our computational result suggested a possible way to analyze the springdamper effect embedded in principle to the biological mechanism for absorbing the ground reaction force flexibly as a common tendency of the walking and running gaits. The analysis with damping factor model by Lankarani \& Nikravesh (1990) realized the fact that a soft grounding effectively reduces the ground reaction force. In the actual phenomenon due to the interaction in the musculoskeletal systems such as a vibration from the sensory feedback loop $[27,28]$, the further detail modeling is crucial. Phenomenologically in the level of the force generation, we successfully observed a time delay in a peak in the force time profile specifically in running gaits, which indicates an intrinsic behavior due to the damping factor is highly important in the kicking motion of the hoof. The phenomenon is also need to be verified with more detail leg models and evidences from the biological system [29,30]. Further considerations are possible to compare in the energy consumption due to the elasticity [18] and robotic application [31]. Geometric characteristics of the hoof is of interest to researchers studying on the biological nature mechanical in the hoof shape and its influence in joint angles and contact timing $[32,33]$.

\section{Acknowledgements}

This work was supported in part by Mongolia-Japan higher Engineering Education Development (MJEED- 
JICA) through the joint research project "Development of industrial, service and intelligent systems based on advanced technologies" (J23A16), JSPS KAKENHI (16H01616, 17H06383) and the New Energy and Industrial Technology Development Organization (NEDO), and Project on Regional Revitalization Through Advanced Robotics (Kyushu Institute of Technology/Kitakyushu city, Japan).

\section{References}

1. D. Joshua Mayich, Alison Novak, Daniel Vena, Timothy R. Daniels, and James W. Brodsky. Gait analysis in orthopedic foot and ankle surgery - Topical review, part 1: Principles and uses of gait analysis. Foot and Ankle International, 35:80-90, 2014.

2. Logan J. Dockery. Analysis of Gait Propulsion During the Ankle and Forefoot Rocker Sub-phases. PhD thesis, 2019.

3. T. Mcgeer. Passive Dynamic Walking. The International Journal of Robotics Research, 9(2):62-82, 1990.

4. P. Mahmoodi, R.S. Ransing, and M.I. Friswell. Modelling the effect of 'heel to toe' rollover contact on the walking dynamics of passive biped robots. Applied Mathematical Modelling, 37(12):7352-7373, 2013.

5. D. Torricelli, J. Gonzalez, M. Weckx, R. Jiménez-Fabián, B. Vanderborght, M. Sartori, S. Dosen, D. Farina, D. Lefeber, and JL. Pons. Human-like compliant locomotion: state of the art of robotic implementations. Bioinspiration and Biomimetics, 11(5), 2016.

6. Eduardo Corral, M. J. Gómez García, Cristina Castejon, Jesús Meneses, and Raúl Gismeros. Dynamic modeling of the dissipative contact and friction forces of a passive biped-walking robot. Applied Sciences (Switzerland), 10, 2020.

7. A. H. Hansen, D. S. Childress, and E. H. Knox. Roll-over shapes of human locomotor systems: effects of walking speed. Clinical biomechanics (Bristol, Avon), 19(4):407414, 2004.

8. A. H. Hansen and D. S. Childress. Investigations of rollover shape: implications for design, alignment, and evaluation of ankle-foot prostheses and orthoses. Disability and Rehabilitation, 32(26):2201-2209, 2010.

9. Bellusci G. Schepers H. M. de Zee M. Andersen M. S. Karatsidis, A. and P. H. Veltink. Estimation of Ground Reaction Forces and Moments During Gait Using Only Inertial Motion Capture. Sensors, 17(1) (75), 2016.

10. Bonnefoy-Mazure A. and Armand S. Normal gait, pages 200-211. Amsterdam: Elsevier, 2015.

11. Alberto Leardini, John J. O'Connor, and Sandro Giannini. Biomechanics of the natural, arthritic, and replaced human ankle joint. Journal of Foot and Ankle Research, 7, 2014.

12. W. Lee and M. Raibert. Control of hoof rolling in an articulated leg. In Proceedings. 1991 IEEE International Conference on Robotics and Automation, pp. 1386-1391.

(C) The 2021 International Conference on Artificial Life and Robotics (ICAROB2021), January 21 to 24, 2021
13. D. Batbaatar and H. Wagatsuma. A proposal of the kinematic model of the horse leg musculoskeletal system by using closed linkages. In IEEE International Conference on Robotics and Biomimetics, pp. 869-874, 2019.

14. T. Jansen. The Great Pretender. Netherlands:010, 2007.

15. K. Komoda and H. Wagatsuma. Energy-efficacy comparisons and multibody dynamics analyses of legged robots with different closed-loop mechanisms. Multibody System Dynamics, 40:123-153, 2017.

16. Sandra D. Starke, Justine J. Robilliard, Renate Weller, Alan M. Wilson, and Thilo Pfau. Walk-run classification of symmetrical gaits in the horse: A multidimensional approach. Journal of the Royal Society Interface, 6:335$342,2009$.

17. Jeffrey J. Thomason and Michael L Peterson. Biomechanical and mechanical investigations of the hooftrack interface in racing horses. Veterinary Clinics: Equine Practice, 24(12):53-77, 2008.

18. R. Mcneill Alexander. Elastic energy stores in running vertebrates. Integrative and Comparative Biology, 24(1):85-94, 1984.

19. J. Baumgarte. Stabilization of constraints and integrals of motion in dynamical systems. Computer Methods in Applied Mechanics and Engineering, 1:1-16, 1972.

20. H. M. Lankarani and P. E. Nikravesh. A contact force model with hysteresis damping for impact analysis of multibody systems. Journal of Mechanical Design, Transactions of the ASME, 112:369-376, 1990.

21. J A C Ambrósio. Impact of Rigid and Flexible Multibody Systems: Deformation Description and Contact Models, pp. 57-81. Springer Netherlands, Dordrecht, 2003.

22. V. P. Chervinskii. Dissipative function of coulomb friction on elliptical contact area. Journal of Friction and Wear, 32:386-393, 2011.

23. Parviz E Nikravesh. Planar multibody dynamics. formulation, programming with Matlab, and applications. CRC Press, second edition, 2019.

24. Gerald Ristow. Simulating granular ow with molecular dynamics. Journal de Physique I, EDP Sciences, 2:649662, 1992.

25. R. McNeill Alexander. Energetics and optimization of human walking and running: The 2000 Raymond Pearl Memorial Lecture. American Journal of Human Biology, 14(5):641-648, 2002.

26. R. McNeill Alexander and A. S. Jayes. Fourier analysis of forces exerted in walking and running. Journal of Biomechanics, 13(4):383-390, 1980.

27. Daniel F. B. Haeufle, Katrin Stollenmaier, Isabelle Heinrich, Syn Schmitt, and Keyan Ghazi-Zahedi. Morphological Computation Increases From Lower- to Higher-Level of Biological Motor Control Hierarchy. Frontiers in Robotics and AI, 7:1-13, 2020. 
28. Z. H. Shen and J. E. Seipel. A fundamental mechanism of legged locomotion with hip torque and leg damping. Bioinspiration and Biomimetics, 7, 2012.

29. Ian Abraham, Zhuohua Shen, and Justin Seipel. A Nonlinear Leg Damping Model for the Prediction of Running Forces and Stability. Journal of Computational and Nonlinear Dynamics, 10:1-8, 2015.

30. Alan M. Wilson, M. Polly McGuigan, Anne Su, and Anton J. Van den Bogert. Horses damp the spring in their step. Nature, 414(6866):895-899, 2001.

31. E. Garcia, J. C. Arevalo, G. Múnoz, and P. Gonzalez-de Santos. On the biomimetic design of agile-robot legs. Sensors, 11:11305-11334, 2011.

32. C. Johnston and W. Back. Hoof ground interaction: When biomechanical stimuli challenge the tissues of the distal limb. Equine Veterinary Journal, 38:634-641, 2006.

33. Angelos Karatsidis, Giovanni Bellusci, H. Martin Schepers, Mark de Zee, Michael S. Andersen, and Peter H. Veltink. Estimation of ground reaction forces and moments during gait using only inertial motion capture. Sensors, 17:1-22, 2017. 